\title{
Carpal tunnel syndrome caused by thrombosed persistent median artery - A case report -
}

Received May 16, 2019

Revised October 1, 2019

Accepted October 2, 2019

\section{Corresponding author}

Sang Yoon Jeon, M.D.

Department of Anesthesiology and

Pain Medicine, Dongkang Medical

Center, 239 Taehwa-ro, Jung-gu, Ulsan

44455, Korea

Tel: 82-52-241-1004

Fax: 82-52-241-1180

E-mail: maestro@paik.ac.kr

\section{Sang Yoon Jeon ${ }^{1}$, Kwangmin Lee ${ }^{2}$, and Weon-Joon Yang ${ }^{2}$}

Department of Anesthesiology and Pain Medicine, 'Dongkang Medical Center, Ulsan, ${ }^{2}$ Cheju Halla General Hospital, Jeju, Korea

Background: A rare case of carpal tunnel syndrome caused by a thrombosed persistent median artery is presented here.

Case: The diagnosis was delayed due to the overlapping cervical radiculopathy. Acute severe pain and nocturnal paresthesia were chief complaints. Ultrasonography, magnetic resonance imaging, and computed tomography angiography revealed that the median nerve was compressed by the occluded median artery. Instead of surgery, conservative therapy was tried. It worked well for six months.

Conclusions: The importance of using modalities for decision making of diagnosis and treatment is emphasized in this report.

Keywords: Carpal tunnel syndrome; Computed tomography angiography; Persistent median artery; Thrombosis.
Carpal tunnel syndrome (CTS) is one of the most common entrapment neuropathies. Its prevalence is $3.8 \%$ [1]. Any condition that constantly compresses the median nerve can impede capillary circulation in the median nerve, leading to ischemia, demyelination, and axonal degeneration [1]. Patients generally present symptoms of pain, nocturnal paresthesia, and numbness in the distribution of the median nerve [1]. In severe cases, motor symptoms with weakness and atrophy of thenar muscles as well as hypesthesia are also shown [1].

The median artery is a transitory artery that forms the arterial axis of the forearm [2]. It generally vanishes by the eighth week of gestation [2]. It occasionally persists in adulthood with a prevalence of $6.6 \%$ [3]. Persistent median artery (PMA) is at risk of thrombosis, leading to CTS [4-6].

In this paper, we report a rare case of carpal tunnel syndrome caused by a thrombosed persistent median artery. We used ultrasonography, magnetic resonance imaging
(MRI), and 3D computed tomography (CT) angiography to diagnose the patient with CTS caused by thrombosis of the PMA and treated him conservatively.

\section{CASE REPORT}

We obtained the patient's verbal consent to publish this case. A 42-year-old man was referred to our pain clinic via the emergency center with a chief complaint of acute severe pain in the right hand. The symptom started three days before he was referred. The pain worsened at night. Numeric rating scale score was 8 over 10 . He was a chassis worker who had been doing a lot of handworks. He had no medical, trauma, or surgery history except a right cervical radiculopathy at the level of $\mathrm{C} 6$. The cervical radiculopathy was diagnosed clinically and radiographically with cervical spine CT 2 weeks before at the neurosurgery clinic. It had caused mild pain and paresthesia from the neck to the

This is an Open Access article distributed under the terms of the Creative Commons Attribution Non-Commercial License (http://creativecommons.org/licenses/by-nc/4.0) which permits unrestricted non-commercial use, distribution, and reproduction in any medium, provided the original work is properly cited.

Copyright (C) the Korean Society of Anesthesiologists, 2020 
right hand (Fig. 1). These symptoms had been somewhat relieved by oral medications (gabapentin, tramadol, acetaminophen). However, when he was referred to our pain clinic, the localized pain in the right hand was aggravated severely and the medication had no effect. On physical examination, he presented no weakness. However, the pain was aggravated by doing palmar flexion of right wrist. We also observed tenderness on the palmaris longus tendon and positive Tinel's sign.

Under the impression of CTS, we investigate it through ultrasonography. In the ultrasonography, we observed that an oval mass was compressing the median nerve which was edematous in the right carpal tunnel (Fig. 2). The mass was scanned from the wrist to the forearm. By doppler image, we

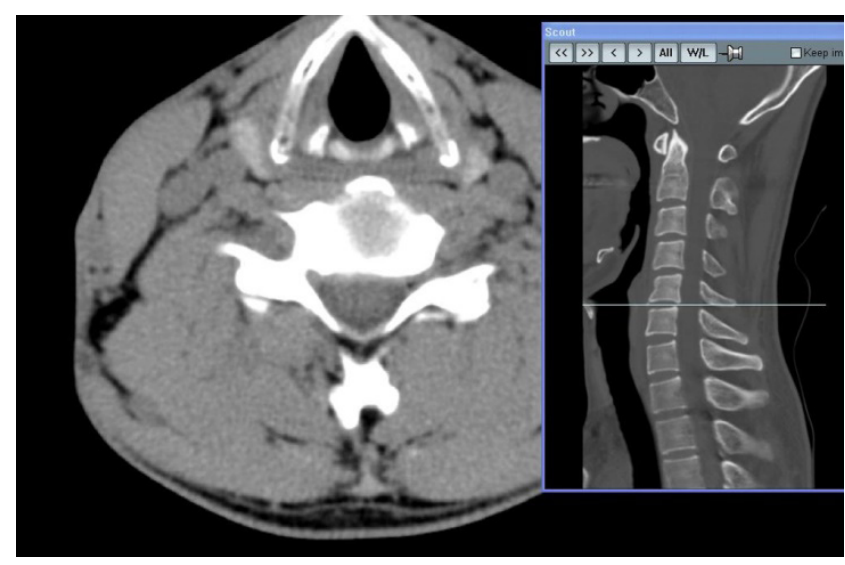

Fig. 1. Transverse and sagittal images at the level of C5-6 of the cervical computed tomography show that the disc is bulging mildly and the right uncovertebral joint is prominent without significant foraminal narrowing.

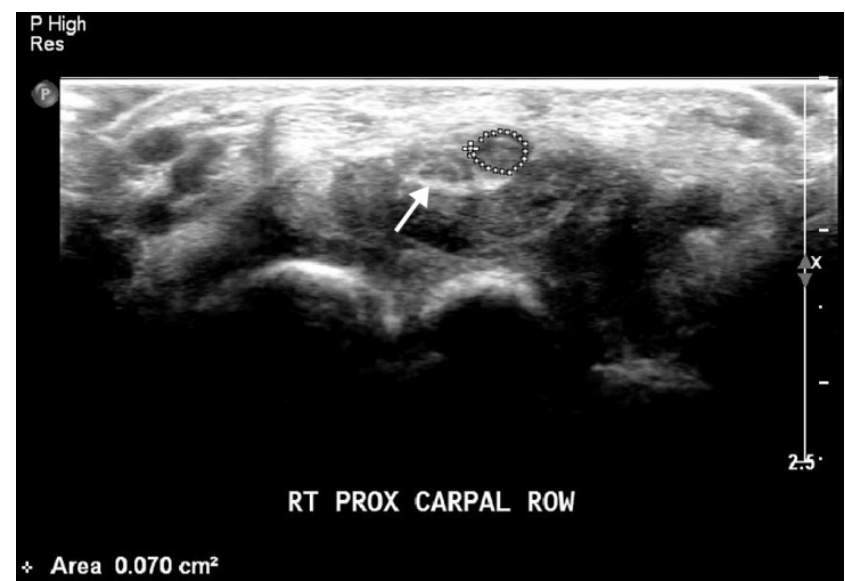

Fig. 2. Ultrasonogram of the thrombosed persistent median artery (dotted circle) and the compressed median nerve (arrow) in the patient's right proximal (prox) carpal tunnel. found that it was the right median artery traveling along with the median nerve from the forearm. It was occluded by hyperechoic thrombosis at the proximal carpal tunnel level (Fig. 3). The patient was firstly diagnosed with CTS. Because he had suspicious lesions in cervical CT and the prescribed oral medication was effective for his symptoms, differential diagnosis was not executed. Thus, the diagnosis of CTS was delayed.

For further imaging study, we performed MRI. During the interval time before taking MRI, although we found thrombosis of the PMA, since he had been diagnosed with cervical radiculopathy, we prescribed him more dose of gabapentin. However, he showed no improvement of the pain. In the MRI, we found that the right median nerve was compressed by a tubular enhancing structure considered as the median artery located at the volar aspect of the median nerve at the level of hook of hamate (Fig. 4B). The median artery was occluded by thrombus. It was relatively large at about $2.7 \mathrm{~mm}$ in diameter at that level. At the level of the pisiform, we found the right median nerve which became edematous (Fig. 4A). Interestingly, the PMA was traveling along with two veins.

After consulting a cardiovascular surgeon considering the need of thrombectomy of the right PMA, we mapped arteries through 3D CT angiography (Fig. 5). In the arteriography, we observed four arteries originated from the right brachial artery. Of these four arteries traveling to the anterior forearm, one remained artery except the ulnar artery, the an-

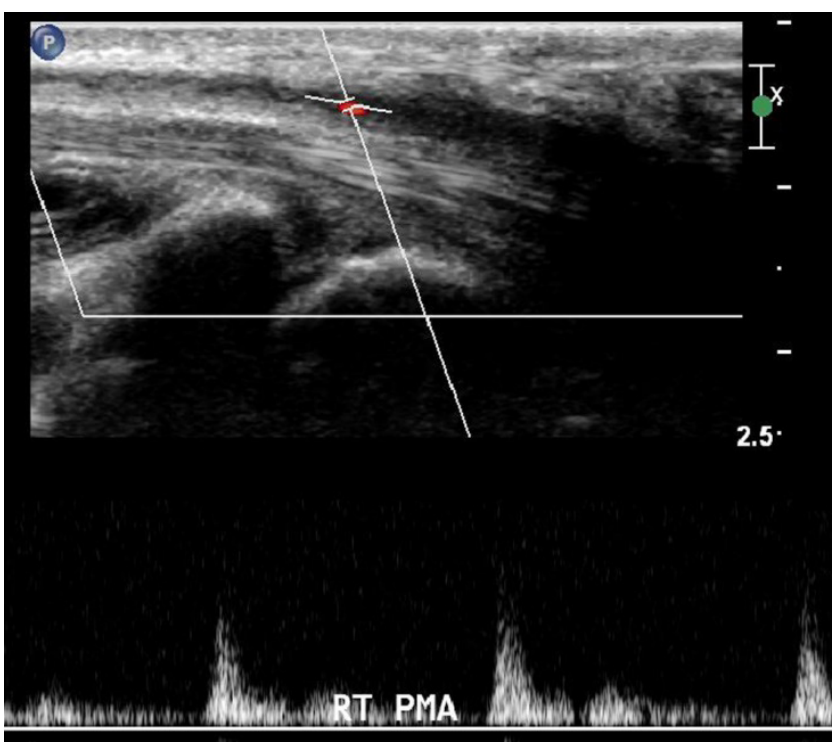

Fig. 3. Longitudinal doppler ultrasonogram of the patient's right persistent median artery (PMA). 
terior interosseous artery, and the posterior interosseous artery was the PMA. The radial artery originated directly from the axillary artery. Moreover, we found that not only the right median artery, but also the left median artery existed. The left median artery was originated from the left brachial artery too. Both were occluded by the thrombus from the distal radioulnar joint level to the metacarpal base level, at which palmar collateral vessels reconstituted the arterial flow of both hands (Fig. 6).

Since there was no ischemic sign in the hand with the help of collateral vessels, we decided to treat the patient conservatively. We prescribed oral antiplatelet drug (cilostazol) and made ultrasound-guided peri-neural steroid injection (triamcinolone $20 \mathrm{mg}$ mixed with $0.1 \%$ ropivacaine $2 \mathrm{ml}$ ) at the right carpal tunnel. After that, his symptoms gradually subsided. They did not recur in one month. The patient was satisfied with the result. He was lost to follow-up.

\section{DISCUSSION}

CTS may be clinically classified into three stages with respect to severity: nocturnal hand pain and paresthesia radiating from wrist to shoulder and being relieved by hand shaking, sensory symptoms during the day and motor deficit, and thenar atrophy and disappearance of sensory symptom [1]. The Katz hand diagrams can help patient localize symptoms in the hand [7]. They are classified into

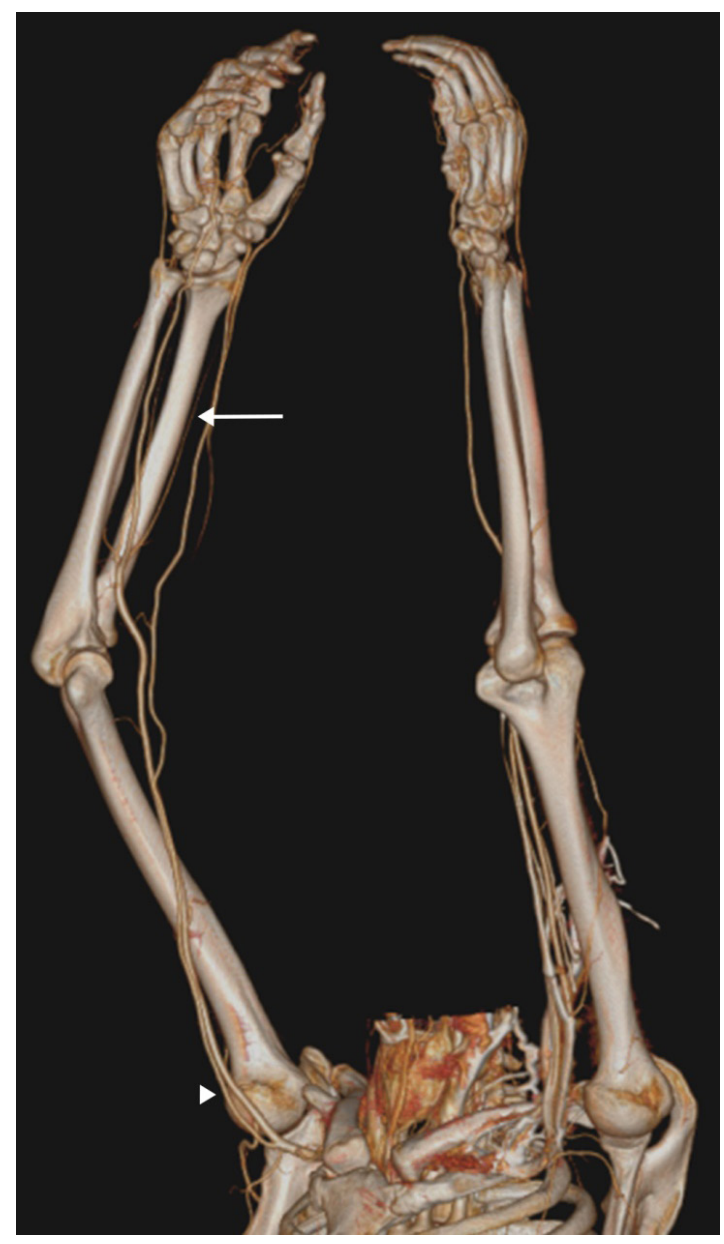

Fig. 5. 3D computed tomography angiogram of the patient's right upper extremity. Each persistent median artery (arrow) and radial artery (arrowhead) are originated from each brachial artery and axillary artery, respectively.
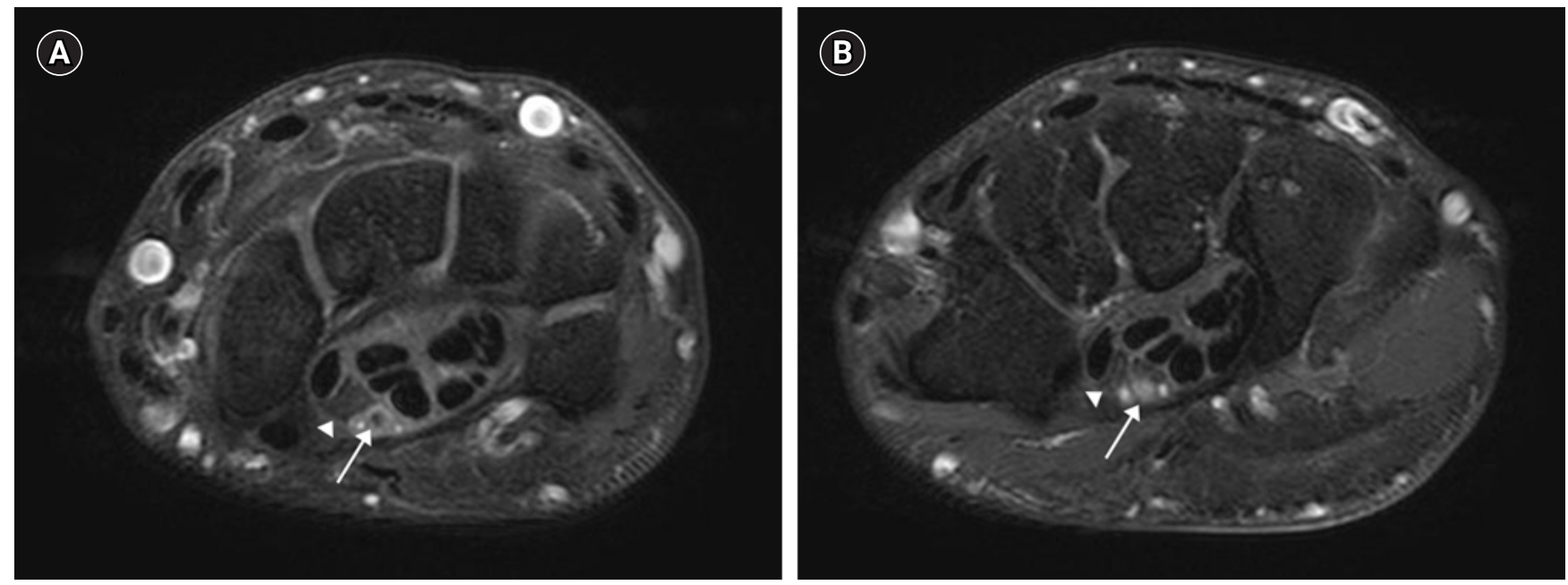

Fig. 4. Persistent median artery in carpal tunnel. Axial gadolinium-enhanced spin-echo T1-weighted magnetic resonance images at levels of pisiform (A) and hook of hamate (B) show persistent median artery (arrow) and two small veins in the carpal tunnel located at volar aspect of median nerve (arrowhead), causing mild impingement of median nerve. 

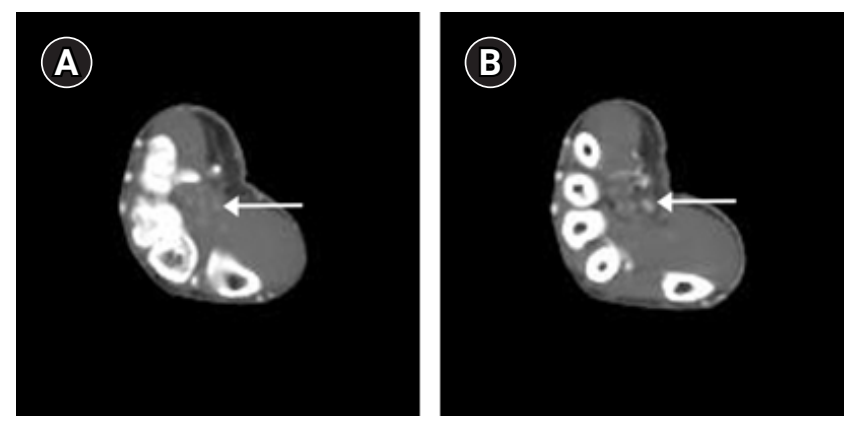

Fig. 6. Computed tomography angiograms of the right upper extremity at levels of hook of hamate $(A)$ in which the persistent median artery (PMA) (arrow) is occluded and base of metacarpal bones $(B)$ in which the PMA's flow is reconstituted by palmar collaterals.

three patterns [7]. Among them, classical or probable pattern is indicative of CTS [7]. According to Keith et al. [8], clinical tests including manual muscle tests and several provocative tests such as Phalen's test, Tinel's test, and median nerve compression test may increase the sensitivity and specificity to diagnose CTS when they are combined together, although each alone has low value for identifying CTS. Therefore, it is required to obtain focused history on symptom onset, pain localization and irradiation, provocative factors, relieving factors, predisposing factors, and working activities [7]. Moreover, combination of symptoms, clinical tests, and electrodiagnostic tests including nerve conduction study and electromyography may be better to diagnose CTS and provide positive correlation with postsurgical outcomes [8].

In fact, the aims of electrodiagnostic tests are to confirm a focal neural damage, to quantify neurophysiological severity, to define the nerve pathophysiology, and to find confounding factors [7]. However, since false negative and false positives can occur, electrodiagnostic tests may confirm CTS, but cannot rule out CTS [1]. Thus, electrodiagnostic tests are recommended when differentiation is required, when thenar atrophy and/or persistent numbness (that is, severe symptoms) are present, and when clinical and/or provocative tests are positive and surgical management is being considered [8]. Ultrasonography has almost the same sensitivity in diagnosing CTS as electrodiagnostic test [1]. It is useful for finding the occupying lesion and determining its severity by measuring cross-sectional area of the median nerve [1]. However, other imaging modalities like MRI are not recommended routinely [1].

CTS must be differentiated from several neuropathies which include cervical radiculopathy, especially $\mathrm{C} 6$ and
C7, brachial plexopathy in upper trunk, proximal median neuropathy, thoracic outlet syndrome, and CNS disorders [1]. When a patient suffers from neuropathic symptoms believed to be caused by cervical radiculopathy, we must not exclude other possible causes. Moreover, if the symptoms are localized to the dermatome of median nerve, differential diagnoses and investigations of peripheral entrapment neuropathies including CTS are required. In comparison with CTS of which the distribution of sensory symptoms ranges between thumb and half of ring finger, $\mathrm{C} 6$ or $\mathrm{C} 7$ cervical radiculopathy has the distribution of sensory symptoms limited to thumb and index finger or middle finger. C6 or C7 cervical radiculopathy might be provoked by Spurling's test and Jackson's compression test [8]. Thus, physical examination should include other areas of the neck and the upper extremity [8].

According to Upton and McComas [9], double crush syndrome (DCS) describes a clinical entity that when a single peripheral nerve is compressed in two or more sites, the first compression leads to disruption of axonal transport along the nerve, thus increasing the vulnerability of distal axons to the second compression. There was also a case of DCS in which the thoracic outlet syndrome was masked by CTS [10]. Thus, the diagnosis was delayed [10]. Therefore, even if a patient is diagnosed to have a certain neuropathy, we should check other confounding factors that may co-exist.

In the present case, both PMAs were found to be occluded by the thrombus. However, the patient experienced acute symptoms in the right hand without symptoms in the left hand. This showed that the occlusion of the PMA might not always cause CTS. We assumed that there might be two factors that caused CTS only in the right hand: the mass effect and the DCS. As we saw in the ultrasonography and the MRI, the occluded right PMA was dilated. That is, high pressure produced by occlusion of the PMA caused luminal dilatation which directly compressed the right median nerve, the so-called mass effect. This effect made the blood supply from collateral vessels to the right median nerve so short that ischemic nerve injury occurred which resulted in edematous status. Moreover, since the patient was diagnosed with right C6 cervical radiculopathy, the proximally compressed root might make the right median nerve weaker to distal compression and poorer blood supply than the left. In fact, it was likely that the mass effect was more dominant than the effect of DCS because the cervical CT images showed mild disc bulging without significant foraminal narrowing and the patient's symptom disappeared after the conservative therapy was done. Ac- 
tually, it would be better if we examined more about cervical radiculopathy with provocative tests for cervical radiculopathy and electrodiagnostic test to measure the effect of the DCS of the cervical radiculopathy and to predict the benefit of surgical procedure which was proposed. On the other hand, although the left PMA was occluded, there was no mass effect. In addition, no DCS existed in the left median nerve. Thus, there was no symptom in the left hand. Therefore, the mass effect was the main mechanism that caused his symptoms.

Although this case showed that thrombosis of the PMA caused CTS, one study reported that the incidence of PMA in a group of CTS patients was 2.4\% [11]. An ipsilateral PMA does not seem to increase the incidence of CTS [11]. However, PMA seems to be associated with anomalies of the median nerve which consists of three forms: a normal median nerve with an eccentric PMA, a bifid median nerve, and high division of the median nerve with an intermediate position of the PMA whose occurrence percentages are $37.5 \%$, $18.75 \%$, and $43.7 \%$, respectively [12]. It was reported that about $8 \%$ of PMAs reached the palm [12]. In some instances, the PMA participated in the formation of the superficial palmar arch supplying blood flow in hands [12]. PMA accompanying veins have also been reported [13].

In our patient, PMAs were not associated with median nerve anomalies. He had a normal right median nerve with an eccentric PMA. We also found some vascular variants, including palmar collaterals extending from each PMA, two accompanying veins along with the right PMA, and high originations of both radial arteries originated from both axillary arteries. With these vascular variants, the fact that he was a chassis worker who frequently used vibrating tools with hands might have promoted the formation of thrombus in the PMAs.

As we incidentally found that his symptoms were caused by occluded PMA by ultrasonography, it was hard to get impression about what the cause was before scanning with ultrasonography. Since CTS caused by occluded PMA is rare, it seems that data about clinical features of CTS caused by occluded PMA distinguished from other causes are insufficient. In a case reported by Dahmam et al. [4], there was a change of temperature in hand, although this was not found in our case or other cases $[5,6]$. This difference may be dependent on the dominance of the median artery of the hand. Its sensitivity may be low because the median artery usually shrinks. However, if a patient complaints CTS accompanying temperature or color changes in hand that may imply vascular disorder, occlusion of PMA might be considered as a possibility.
Patients with CTS can be treated by conservative cares including local and systemic steroids, vitamins B6 and B12, non-steroidal anti-inflammatory drugs, ultrasound, yoga, carpal bone mobilization and wrist splints, and surgical treatment in the form of carpal tunnel release [1]. When patients suffer from severe acute symptoms caused by occluded PMAs, some reports recommend surgery $[4,5]$. In another report, when collateral vessels exist, conservative therapy using anti-coagulants was successful [6]. Since we confirmed the collateral's existence by CT angiography without observing ischemic signs, we tried conservative treatment which worked well.

Since acute CTS is unusual without trauma, investigating the etiology by imaging modality is recommended. In the present case, using ultrasonography, we could diagnose CTS and find the occluded PMA as the cause simultaneously and promptly. However, we could not acquire the exact lesion of the compressed median nerve or the occluded PMA because of the low resolution of ultrasonography. With the aid of MRI, it was possible to obtain the exact anatomy of the patient's wrist which might have had an anomaly. MRI also revealed the accurate lesion and the severity of the median nerve compressed which helped us decide on the management and predict the benefit from surgical intervention. 3D CT angiography provided us the information about variants of arteries and existence of collateral vessels which was also helpful for deciding management. It would have been useful for surgery to reduce surgical and iatrogenic injuries.

In conclusion, when a patient who is suspected to have acute CTS comes to clinic, despite low incidence of PMA, it is important to investigate the existence of PMA and make differential diagnosis. When a thrombosed PMA is found to be the cause of CTS, if there are collateral vessels without signs of ischemia, conservative therapy instead of surgery may be tried to treat the patient.

\section{CONFLICTS OF INTEREST}

No potential conflict of interest relevant to this article was reported.

\section{AUTHOR CONTRIBUTIONS}

Conceptualization: Sang Yoon Jeon. Data acquisition: Sang Yoon Jeon. Formal analysis: Sang Yoon Jeon. Supervision: Weon-Joon Yang. Writing_original draft: Kwangmin 
Lee. Writing_review \& editing: Kwangmin Lee, Sang Yoon Jeon, Weon-Joon Yang.

\section{ORCID}

Sang Yoon Jeon, https://orcid.org/0000-0003-1251-1396

Kwangmin Lee, https://orcid.org/0000-0003-4061-9740

Weon-Joon Yang, https://orcid.org/0000-0001-6505-9490

\section{REFERENCES}

1. Ibrahim I, Khan WS, Goddard N, Smitham P. Carpal tunnel syndrome: a review of the recent literature. Open Orthop J 2012; 6: 69-76.

2. Kopuz C, Baris S, Gulman B. A further morphological study of the persistent median artery in neonatal cadavers. Surg Radiol Anat 1997; 19: 403-6.

3. Singla RK, Kaur N, Dhiraj GS. Prevalence of the persistant median artery. J Clin Diagn Res 2012; 6: 1454-7.

4. Dahmam A, Matter-Parrat V, Manguila F, Giannikas D, Marin Braun F. Acute carpal tunnel syndrome due to a thrombosed persistent median artery: unusual cause in athletes. J Traumatol Sport 2015; 32: 126-8.

5. Kele H, Verheggen R, Reimers CD. Carpal tunnel syndrome caused by thrombosis of the median artery: the importance of high-resolution ultrasonography for diagnosis. Case report. J Neurosurg 2002; 97: 471-3.
6. Srivastava A, Sharma P, Pillay S. Persistent median artery thrombosis: a rare cause of carpal tunnel syndrome. Australas J Ultrasound Med 2015; 18: 82-5.

7. Alfonso C, Jann S, Massa R, Torreggiani A. Diagnosis, treatment and follow-up of the carpal tunnel syndrome: a review. Neurol Sci 2010; 31: 243-52.

8. Keith MW, Masear V, Chung K, Maupin K, Andary M, Amadio PC, et al. Diagnosis of carpal tunnel syndrome. J Am Acad Orthop Surg 2009; 17: 389-96

9. Upton AR, McComas AJ. The double crush in nerve entrapment syndromes. Lancet 1973; 2: 359-62.

10. Seok JH, Lee JH, Sim KS, Ban JS, Lee JH, Kim EJ. Overlapped multiple distal entrapment neuropathies hindering diagnosis of thoracic outlet syndrome -a case report-. Anesth Pain Med 2012; 7: 348-51.

11. Altinkaya N, Leblebici B. Prevalence of persistent median artery in carpal tunnel syndrome: sonographic assessment. Surg Radiol Anat 2016; 38: 511-5

12. Gassner EM, Schocke M, Peer S, Schwabegger A, Jaschke W, Bodner G. Persistent median artery in the carpal tunnel: color Doppler ultrasonographic findings. J Ultrasound Med 2002; 21: 455-61.

13. Feintisch AM, Ayyala HS, Datiashvili R. An anatomic variant of persistent median artery in association with carpal tunnel syndrome: case report and review of the literature. J Hand Surg Asian Pac Vol 2017; 22: 523-5. 